\section{Annual Report of the University of Kansas Health System Poison Control Center}

Lisa K. Oller, R.Ph., Doyle M. Coons, BSN, Stephen L. Thornton, M.D.

University of Kansas Health System Poison Control Center, Kansas City, KS

Received March 14, 2019; Accepted for publication April 1, 2019; Published online Aug. 21, 2019

\section{ABSTRACT}

Introduction. This is the 2017 Annual Report of the University of Kansas Health System Poison Control Center (PCC). The PCC is one of 55 certified poison control centers in the United States and serves the state of Kansas 24-hours a day, 365 days a year. The PCC receives calls from the public, law enforcement, health care professionals, and public health agencies, which are answered by trained and certified specialists in poison information with the immediate availability of medical toxicology back up. All calls to the PCC are recorded electronically in the Toxicall ${ }^{\varpi}$ data management system and uploaded in near real-time to the National Poison Data System (NPDS), which is the data repository for all poison control centers in the United States.

Methods. All encounters reported to the PCC from January 1, 2017 to December 31, 2017 were analyzed. Data recorded for each exposure included caller location, age, weight, gender, substance exposed to, nature of exposure, route of exposure, interventions, medical outcome, disposition and location of care. Encounters were classified further as human exposure, animal exposure, confirmed non-exposure, or information call (no exposure reported).

Results. The PCC logged 21,431 total encounters in 2017, including 20,278 human exposure cases. Cases came from every county in Kansas. Most of the human exposure cases $(51.4 \%, n=10,430)$ were female. Approximately $66 \%(\mathrm{n}=13,418)$ of human exposures involved a child (defined as age less than 20 years). Most encounters occurred at a residence $(94.0 \%, \mathrm{n}=19,018)$ and most calls $(69.5 \%, \mathrm{n}$ $=14,090)$ originated from a residence. Almost all human exposures ( $\mathrm{n}=19,823$ ) were acute cases (exposures occurring over eight hours or less). Ingestion was the most common route of exposure documented $(80.5 \%, \mathrm{n}=17,466)$. The most common reported substance in pediatric encounters was cosmetics/personal care products ( $\mathrm{n}$ $=1,255)$, followed by household cleaning products $(\mathrm{n}=1,251)$. For adult encounters, analgesics $(\mathrm{n}=1,160)$ and sedatives/hypnotics/ antipsychotics $(n=1,127)$ were the most frequently involved substances. Unintentional exposures were the most common reason for exposures $(78.6 \%, \mathrm{n}=15,947)$. Most encounters $(69.4 \%, \mathrm{n}=$ $14,073)$ were managed in a non-health care facility (i.e., a residence). Among human exposures, 14,940 involved exposures to pharmaceutical agents, while 9,896 involved exposure to non-pharmaceuticals. Medical outcomes were 28\% ( $\mathrm{n}=5,741)$ no effect, $18 \%(\mathrm{n}=3,693)$ minor effect, $9 \%(n=1,739)$ moderate effect, and $2 \%(n=431)$ major effect. There were 16 deaths in 2017 reported to the PCC. Number of exposures, calls from health care facilities, cases with moderate or
KANSAS JOURNAL of MEDIC INE

major medical outcomes, and deaths increased in 2017 compared to 2016, despite a decrease in total exposures.

Conclusions. The results of the 2017 University of Kansas Health System Poison Control annual report demonstrated that the center continues to receive calls from the entire state of Kansas, totaling over 20,000 human exposures per year. While pediatric exposures remain the most common, a trend of increasing number of calls remains from health care facilities and for cases with serious outcomes. The 2017 PCC data reflected current national trends. This report demonstrated the continued importance of the PCC to both the public and health care providers in the state of Kansas.

\section{Kans J Med 2019;12(3):70-79.}

\section{INTRODUCTION}

This is the 2017 Annual Report of University of Kansas Health System Poison Control Center (PCC). The PCC is a 24-hour 365 day/year health care information resource serving the state of Kansas. It was founded in 1982 and is certified with the American Association of Poison Control Centers (AAPCC). There are 55 certified poison control centers in the United States. The PCC is staffed by nine certified specialists in poison information who are either critical care trained nurses or doctors of pharmacy. There is 24-hour back-up provided by four fellowship trained, board certified medical toxicologists.

The PCC receives calls from the public, law enforcement, health care professionals, and public health agencies. Encounters may involve an exposed animal or human (Exposure Call) or a request for information with no known exposure (Information Call). The PCC follows all cases to make management recommendations, monitor case progress, and document medical outcome. This information is recorded electronically in the Toxicall $^{\circledast}$ data management system and uploaded in near real-time to the National Poison Data System (NPDS). NPDS is the data warehouse for all the nation's poison control centers. ${ }^{1}$ The average time to upload data for all PCCs is 9.50 [7.33, 14.6] (median [25\%, 75\%]) minutes, creating a real-time national exposure database and surveillance system. The PCC has the ability to share NPDS real time surveillance with state and local health departments and other regulatory agencies. An analysis and summary of all encounters reported to the PCC from January 1, 2017 to December 31, 2017 follows.

\section{METHODS}

All PCC encounters recorded electronically in the Toxicall ${ }^{\oplus}$ data management system from January 1, 2017 to December 31, 2017 were analyzed. Cases were first classified as either an exposure or suspected exposure (Human Exposure, Animal Exposure, Non-Exposure Confirmed Cases) or a request for information with no reported exposure (Information Call). Extracted data included caller location, age, weight, gender, exposure substance, number of follow-up calls, nature of exposure (i.e., unintentional, recreational, or intentional), exposure scenario, route of exposure (oral, dermal, parenteral), 


\section{KANSAS JOURNAL of MEDICINE}

$20 I 7$ POISON CONTROL CENTER REPORT

continued.

interventions, medical outcome (no effect, minor, moderate, severe or death), disposition (admitted to noncritical care unit, admitted to critical care unit, admitted to psychiatry unit, lost to follow-up or treated and released), and location of care (non-health care facility or health care facility). For this analysis, a pediatric case was defined as any patient 19 years of age or less. This is consistent with NPDS methodology. For medical outcome, the following definitions were used: minor (minimally bothersome symptoms), moderate (more pronounced symptoms, usually requiring treatment), and major (life threatening signs and symptoms).

Data were analyzed using Microsoft Excel (Microsoft Corp, Redmond, WA).

\section{RESULTS}

The PCC logged 21,431 total calls in 2017, including 20,278 human exposure cases, 77 non-exposure confirmed cases, 119 animal exposure cases, and 957 information calls. This was a decrease of 534 calls $(2.4 \%)$ compared to 2016 . For information calls, drug information $(\mathrm{n}=311)$ was most common reason for calling. Table 1 further describes the encounter types. The PCC made 31,715 follow-up calls in 2017. Follow-up calls were done in $58.8 \%$ of human exposure cases. One follow-up call was made in $26.9 \%$ of human exposure cases; multiple follow-up calls (range 2 - 45) were made in $32.0 \%$ of cases. In human exposure calls for which follow-up calls were made, an average of 2.66 follow-up calls per case were performed.

The PCC received calls from all 105 counties in Kansas. The county with the largest number of calls was Sedgwick County with 3,260. In addition, calls were received from 48 states, the District of Columbia, Puerto Rico, and the US Virgin Islands; five calls were from foreign countries.

The majority of human exposure cases $(51.4 \%, \mathrm{n}=10,430)$ were female. A male predominance was found among encounters involving children younger than 13 years of age, but this gender distribution was reversed in teenagers and adults, with females comprising most of the reported exposures. Approximately 66\% $(n=13,418)$ of human exposures involved a child (defined as age 19 years or less). Table 2 illustrates distribution of human exposures by age and gender. Patients one year of age were the most common age group involved in encounters reported to the PCC. For adults, the age group of 20 - 29 years old was most commonly encountered. Seventy-seven (77) exposures occurred in pregnant women $(0.4 \%$ of all human exposures). Of these exposures, $29.9 \%$ occurred in the first trimester, $37.7 \%$ occurred in the second trimester, and $27.3 \%$ occurred in the third trimester. Most of these exposures (71.4\%) were unintentional exposures and $19.5 \%$ were intentional exposures. There were no reported deaths to PCC in pregnant women in 2017.
Table 1. Encounter type.

\begin{tabular}{|l|c|c|}
\hline & Number & $\%$ \\
\hline Exposure & & \\
\hline Human exposure & 20,278 & 94.32 \\
\hline Animal exposure & 119 & 0.51 \\
\hline \multicolumn{1}{|c|}{ Subtotal } & $\mathbf{2 0 , 3 9 7}$ & $\mathbf{9 4 . 8 3}$ \\
\hline Non-Exposure Confirmed Cases & & \\
\hline Human non-exposure & 77 & 0.39 \\
\hline \multicolumn{1}{|c|}{ Subtotal } & $\mathbf{7 7}$ & $\mathbf{0 . 3 9}$ \\
\hline Information Call & & \\
\hline Drug information & 311 & 1.40 \\
\hline Drug identification & 117 & 0.86 \\
\hline Environmental information & 94 & 0.56 \\
\hline Medical information & 14 & 0.14 \\
\hline Occupational information & 5 & 0.00 \\
\hline Poison information & 112 & 0.50 \\
\hline Prevention/safety/education & 19 & 0.14 \\
\hline Teratogenicity information & 4 & 0.00 \\
\hline Other information & 46 & 0.22 \\
\hline Substance abuse & 11 & 0.03 \\
\hline Administrative & 6 & 0.07 \\
\hline Caller referred & 218 & 0.86 \\
\hline & $\mathbf{9 5 7}$ & $\mathbf{4 . 7 8}$ \\
\hline Total & $\mathbf{2 1 , 4 3 1}$ & $\mathbf{1 0 0 . 0 0}$ \\
\hline
\end{tabular}

For human exposures, 69.5\% $(\mathrm{n}=14,090)$ of calls originated from a residence (own or other), while 93.8\% ( $n=19,018)$ of these exposures actually occurred at a residence (own or other). Calls from a health care facility accounted for $24.1 \%(n=4,892)$ of human exposure encounters. Table 3 further details the origin of human exposure calls and where the exposure took place.

Human exposures were predominantly $(87.3 \%, \mathrm{n}=17,694)$ acute cases (i.e., exposures occurring over eight hours or less). Chronic exposures (exposures occurring more than eight hours) accounted for $1.8 \%$ (365) of all human exposures reported. Acute or chronic exposures (single exposure that was preceded by a chronic exposure of more than eight hours) totaled 2,129 (10.5\%). Ingestion was the most common route of exposure documented $(80.5 \%, \mathrm{n}=17,466$; Table 4) in all cases.

The most common reported substance in those less than five years of age was cosmetics/personal care products $(n=1,255)$ followed closely by household cleaning products $(\mathrm{n}=1,251)$. For adult $(>19$ years of age) encounters, analgesics $(n=1,160)$ and sedatives/hypnotics/antipsychotics $(n=1,127)$ were the most frequently involved substances. Among all encounters, analgesics $(n=2,833,11.3 \%)$ were the most frequently encountered substance category. Table 5 lists most frequently encountered substance categories for pediatric encounters and Table 6 lists those for adult encounters. A summary $\log$ for all exposures categorized by category and sub-category of substance is presented in an online supplemental appendix (journals. ku.edu/kjm). 
KANSAS JOURNAL of MEDICINE

2017 POISON CONTROL CENTER REPORT

continued.

Table 2. Distribution of human exposures by age and gender.

\begin{tabular}{|c|c|c|c|c|c|c|c|c|c|c|}
\hline & \multicolumn{2}{|c|}{ Male } & \multicolumn{2}{|c|}{ Female } & \multicolumn{2}{|c|}{ Unknown Gender } & \multicolumn{2}{|c|}{ Total } & \multicolumn{2}{|c|}{ Cumulative Total } \\
\hline Age & $\mathbf{N}$ & $\begin{array}{c}\text { \% of Age } \\
\text { Group } \\
\text { Total }\end{array}$ & $\mathbf{N}$ & $\begin{array}{c}\% \text { of Age } \\
\text { Group } \\
\text { Total }\end{array}$ & $\mathbf{N}$ & $\begin{array}{c}\% \text { of Age } \\
\text { Group } \\
\text { Total }\end{array}$ & $\mathbf{N}$ & $\begin{array}{c}\% \text { of } \\
\text { Total } \\
\text { Exposure }\end{array}$ & $\mathbf{N}$ & $\%$ \\
\hline$<$ l year & 584 & 51.64 & 545 & 48.19 & 2 & 0.18 & 1,131 & 5.58 & 1,131 & 5.58 \\
\hline 1 year & 1,792 & 53.18 & 1,577 & 46.80 & 1 & 0.03 & 3,370 & 16.62 & 4,501 & 22.20 \\
\hline 2 years & 1,734 & 52.91 & 1,540 & 46.99 & 3 & 0.09 & 3,277 & 16.16 & 7,778 & 38.36 \\
\hline 3 years & 809 & 54.66 & 671 & 45.34 & 0 & 0.00 & 1,480 & 7.30 & 9,258 & 45.66 \\
\hline 4 years & 400 & 56.58 & 307 & 43.42 & 0 & 0.00 & 707 & 3.49 & 9,965 & 49.14 \\
\hline 5 years & 274 & 62.70 & 163 & 37.30 & 0 & 0.00 & 437 & 2.16 & 10,402 & 51.30 \\
\hline Unknown $\leq 5$ years & 3 & 100.00 & 0 & 0.00 & 0 & 0.00 & 3 & 0.01 & 10,405 & 51.31 \\
\hline Child $6-12$ years & 702 & 59.90 & 469 & 40.02 & 1 & 0.09 & 1,172 & 5.78 & 11,577 & 57.09 \\
\hline Teen 13 - 19 years & 645 & 35.19 & 1,186 & 64.70 & 2 & 0.11 & 1,833 & 9.04 & 13,410 & 66.13 \\
\hline Unknown child & 4 & 50.00 & 3 & 37.50 & 1 & 12.50 & 8 & 0.04 & 13,418 & 66.17 \\
\hline Subtotal & 6,947 & 51.77 & 6,461 & 48.15 & 10 & 0.07 & 13,418 & 66.17 & 13,418 & 66.17 \\
\hline $20-29$ years & 808 & 45.27 & 977 & 54.73 & 0 & 0.00 & 1,785 & 8.80 & 15,203 & 74.97 \\
\hline $30-39$ years & 612 & 43.37 & 799 & 56.63 & 0 & 0.00 & 1,411 & 6.96 & 16,614 & 81.93 \\
\hline $40-49$ years & 411 & 38.48 & 657 & 61.52 & 0 & 0.00 & 1,068 & 5.27 & 17,682 & 87.20 \\
\hline $50-59$ years & 387 & 40.95 & 558 & 59.05 & 0 & 0.00 & 945 & 4.66 & 18,627 & 91.86 \\
\hline $60-69$ years & 316 & 42.93 & 419 & 56.93 & 1 & 0.14 & 736 & 3.63 & 19,363 & 95.49 \\
\hline 70 - 79 years & 175 & 37.88 & 287 & 62.12 & 0 & 0.00 & 462 & 2.28 & 19,825 & 97.77 \\
\hline 80 - 89 years & 80 & 32.13 & 169 & 67.87 & 0 & 0.00 & 249 & 1.23 & 20,074 & 98.99 \\
\hline$\geq 90$ years & 24 & 38.71 & 38 & 61.29 & 0 & 0.00 & 62 & 0.31 & 20,136 & 99.30 \\
\hline Unknown adult & 45 & 47.87 & 49 & 52.13 & 0 & 0.00 & 94 & 0.46 & 20,230 & 99.76 \\
\hline Subtotal & 2,858 & 41.96 & 3,953 & 58.03 & 1 & 0.01 & 6,812 & 33.59 & 20,230 & 99.76 \\
\hline Total* & 9,812 & 48.39 & 10,430 & 51.44 & 36 & 0.18 & 20,278 & 100.00 & 20,278 & 100.00 \\
\hline
\end{tabular}

*Total includes 48 unknown age cases.

Table 3. Origin of call and site of exposure for human exposure cases.

\begin{tabular}{|l|c|c|c|c|}
\hline \multicolumn{1}{|c|}{ Site } & \multicolumn{2}{c|}{ Origin of Call } & \multicolumn{2}{c|}{ Site of Exposure } \\
\hline & $\mathbf{N}$ & $\boldsymbol{\%}$ & $\mathbf{N}$ & $\%$ \\
\hline Residence & & & & \\
\hline \multicolumn{1}{|c|}{ Other } & 13,773 & 67.92 & 18,315 & 90.32 \\
\hline Workplace & 317 & 1.56 & 703 & 3.47 \\
\hline Health care facility & 269 & 1.33 & 438 & 2.16 \\
\hline School & 4,892 & 24.12 & 77 & 0.38 \\
\hline Restaurant/food service & 52 & 0.26 & 221 & 1.09 \\
\hline Public area & 4 & 0.02 & 36 & 0.18 \\
\hline Other & 70 & 0.35 & 175 & 0.86 \\
\hline Unknown & 889 & 4.38 & 227 & 1.12 \\
\hline
\end{tabular}


KANSAS JOURNAL of MEDICINE

2017 POISON CONTROL CENTER REPORT

continued.

Table 4. Route of human exposures.

\begin{tabular}{|l|c|c|c|}
\hline & \multicolumn{3}{|c|}{ Human Exposures } \\
\hline Route & $\mathbf{N}$ & \% of All Routes & \% of All Cases \\
\hline Ingestion & 17,466 & 80.54 & 86.13 \\
\hline Dermal & 1,663 & 7.67 & 8.20 \\
\hline Inhalation/nasal & 1,159 & 5.34 & 5.72 \\
\hline Ocular & 820 & 3.78 & 4.04 \\
\hline Bite/sting & 203 & 0.94 & 1.00 \\
\hline Unknown & 168 & 0.77 & 0.83 \\
\hline Parenteral & 148 & 0.68 & 0.73 \\
\hline Other & 23 & 0.11 & 0.11 \\
\hline Otic & 22 & 0.10 & 0.11 \\
\hline Rectal & 7 & 0.03 & 0.03 \\
\hline Aspiration (with ingestion) & 4 & 0.02 & 0.02 \\
\hline Vaginal & 4 & 0.02 & 0.02 \\
\hline Total number of routes & $\mathbf{2 1 , 6 8 7}$ & $\mathbf{1 0 0 . 0 0}$ & $\mathbf{1 0 6 . 9 5}$ \\
\hline
\end{tabular}

*Some cases may have multiple routes of exposure documented.

Table 5. Substance categories most frequently involved in exposures for ages five years or less.

\begin{tabular}{|l|c|c|c|c|}
\hline \multicolumn{1}{|c|}{ Substance Category } & $\begin{array}{c}\text { All } \\
\text { Substance }\end{array}$ & $\%$ & $\begin{array}{c}\text { Single } \\
\text { Substance } \\
\text { Exposures }\end{array}$ & $\%$ \\
\hline $\begin{array}{l}\text { Cosmetics/personal care } \\
\text { products }\end{array}$ & 1,255 & 11.48 & 1,221 & 12.16 \\
\hline $\begin{array}{l}\text { Cleaning substances } \\
\text { (household) }\end{array}$ & 1,251 & 11.44 & 1,204 & 11.99 \\
\hline Analgesics & 996 & 9.11 & 886 & 8.82 \\
\hline Antihistamines & 607 & 5.55 & 543 & 5.41 \\
\hline $\begin{array}{l}\text { Foreign bodies/toys/ } \\
\text { miscellaneous }\end{array}$ & 541 & 4.95 & 523 & 5.21 \\
\hline Topical preparations & 534 & 4.88 & 514 & 5.12 \\
\hline Vitamins & 468 & 4.28 & 417 & 4.15 \\
\hline Pesticides & 429 & 3.92 & 417 & 4.15 \\
\hline $\begin{array}{l}\text { Dietary supplements/herbals/ } \\
\text { homeopathic }\end{array}$ & 409 & 3.74 & 378 & 3.76 \\
\hline Gastrointestinal preparations & 292 & 2.67 & 257 & 2.56 \\
\hline Plants & 275 & 2.51 & 268 & 2.67 \\
\hline Antimicrobials & 237 & 2.17 & 217 & 2.16 \\
\hline Electrolytes and minerals & 231 & 2.11 & 205 & 2.04 \\
\hline Cold and cough preparations & 222 & 2.03 & 199 & 1.98 \\
\hline $\begin{array}{l}\text { Hormones and hormone } \\
\text { antagonists }\end{array}$ & 202 & 1.85 & 156 & 1.55 \\
\hline
\end{tabular}

Table 6. Substance categories most frequently involved in exposures of adults ( $>19$ years).

\begin{tabular}{|l|c|c|c|c|}
\hline \multicolumn{1}{|c|}{ Substance Category } & $\begin{array}{c}\text { All } \\
\text { Substance }\end{array}$ & $\%$ & $\begin{array}{c}\text { Single } \\
\text { Substance } \\
\text { Exposures }\end{array}$ & $\%$ \\
\hline Analgesics & 1,160 & 11.45 & 489 & 9.55 \\
\hline $\begin{array}{l}\text { Sedative/hypnotics/ } \\
\text { antipsychotics }\end{array}$ & 1,127 & 11.12 & 357 & 6.97 \\
\hline Antidepressants & 848 & 8.37 & 274 & 5.35 \\
\hline Cardiovascular drugs & 713 & 7.04 & 231 & 4.51 \\
\hline Alcohols & 482 & 4.76 & 56 & 1.09 \\
\hline $\begin{array}{l}\text { Cleaning substances } \\
\text { (household) }\end{array}$ & 437 & 4.31 & 342 & 6.68 \\
\hline Anticonvulsants & 427 & 4.21 & 123 & 2.40 \\
\hline Antihistamines & 416 & 4.10 & 179 & 3.49 \\
\hline Pesticides & 397 & 3.92 & 153 & 2.99 \\
\hline $\begin{array}{l}\text { Hormones and hormone } \\
\text { antagonists }\end{array}$ & 308 & 3.04 & 153 & 2.99 \\
\hline Stimulants and street drugs & 295 & 2.91 & 106 & 2.07 \\
\hline Fumes/gases/vapors & 230 & 2.27 & 206 & 4.02 \\
\hline Chemicals & 223 & 2.20 & 186 & 3.63 \\
\hline $\begin{array}{l}\text { Cosmetics/personal care } \\
\text { products }\end{array}$ & 216 & 2.13 & 187 & 3.65 \\
\hline Muscle relaxants & 211 & 2.08 & 76 & 1.48 \\
\hline
\end{tabular}

There was a total of 395 plant exposures reported to the PCC. The most common plant exposure encountered was to pokeweed (Phytolacca americana) $(\mathrm{n}=45)$. Table 7 lists the top five most encountered plants.

Table 7. Top five most frequent plant exposures.

\begin{tabular}{|l|c|}
\hline Botanical Name or Category & N \\
\hline Phytolacca americana (L.) (Pokeweed) & 45 \\
\hline Cherry (Species unspecified) & 18 \\
\hline Plants - Toxicodendrol & 16 \\
\hline Philodendron (Species unspecified) & 13 \\
\hline Spathiphyllum species (Botanic name) & 10 \\
\hline Unknown plant & 28 \\
\hline
\end{tabular}

Unintentional exposures were the most common reason for exposures $(78.6 \%, \mathrm{n}=15,947)$ while intentional exposures accounted for $18.8 \%(n=3,818)$ of exposures. Compared to 2016 , there was a $12.5 \%$ increase in the number of intentional exposures $(n=441)$. Table 8 lists reasons for human exposures. Most unintentional exposures, $65.0 \%(n=10,361)$, occurred in the less than five years old age group. Up to 12 years of age, $98.5 \%(n=11,577)$ of ingestions were unintentional. However, in the $13-19$ age group, intentional exposure was most common $(67 \%, \mathrm{n}=1,229)$. In total, suspected suicide attempts accounted for $14.3 \%(n=2,906)$ of human encounters. This was an increase of $17 \%(n=491)$ compared to 2016 data. When a therapeutic error was the reason for exposure, a double dose was the most common scenario, $29.4 \%(n=727)$. 
Table 8. Reason for human exposure cases.

\begin{tabular}{|c|c|c|}
\hline Reason & $\mathbf{N}$ & \% Human Exposures \\
\hline \multicolumn{3}{|l|}{ Unintentional } \\
\hline General & 10,847 & 53.5 \\
\hline Therapeutic error & 2,395 & 11.8 \\
\hline Misuse & 1,371 & 6.8 \\
\hline Environmental & 596 & 2.9 \\
\hline Occupational & 345 & 1.7 \\
\hline Bite/sting & 206 & 1.0 \\
\hline Food poisoning & 165 & 0.8 \\
\hline Unknown & 22 & 0.1 \\
\hline Subtotal & 15,947 & 78.6 \\
\hline \multicolumn{3}{|l|}{ Intentional } \\
\hline Suspected suicide & 2,906 & 14.3 \\
\hline Misuse & 486 & 2.4 \\
\hline Abuse & 335 & 1.7 \\
\hline Unknown & 91 & 0.4 \\
\hline Subtotal & 3,818 & 18.8 \\
\hline \multicolumn{3}{|l|}{ Adverse reaction } \\
\hline Drug & 241 & 1.2 \\
\hline Other & 65 & 0.3 \\
\hline Food & 37 & 0.2 \\
\hline Subtotal & 343 & 1.7 \\
\hline \multicolumn{3}{|l|}{ Unknown } \\
\hline Unknown reason & 89 & 0.4 \\
\hline Subtotal & 89 & 0.4 \\
\hline \multicolumn{3}{|l|}{ Other } \\
\hline Malicious & 56 & 0.3 \\
\hline $\begin{array}{l}\text { Contamination/ } \\
\text { tampering }\end{array}$ & 15 & 0.1 \\
\hline Withdrawal & 10 & 0.0 \\
\hline Subtotal & 81 & 0.4 \\
\hline Total & 20,278 & 100.0 \\
\hline
\end{tabular}

Most encounters $(69.4 \%, \mathrm{n}=14,073)$ were managed in a non-health care facility (i.e., a residence). Of the 5,982 encounters managed at a health care facility, $47.7 \%(\mathrm{n}=2,851)$ were admitted. Table 9 lists the management site of all human encounters.

Among human exposures, 14,940 involved exposures to pharmaceutical agents while 9,896 involved exposure to non-pharmaceuticals. Because an encounter could include numerous pharmaceutical and non-pharmaceutical agents, this total is greater than the total number of encounters. However, 87.3\% $(n=17,700)$ of all human exposures were exposed to only a single substance. Among these single substance exposures, the reason for exposure was intentional in $22.2 \%$ $(n=1,980)$ of pharmaceutical-only cases compared to $3.6 \%(n=319)$ of non-pharmaceutical single substance exposures.

When medical outcomes were analyzed, $28.3 \%(n=5,741)$ of human exposures had no effect, $18.2 \%(\mathrm{n}=3,693)$ had minor effect, $8.5 \%(n=1,739)$ had moderate effect, and $2.1 \%(n=431)$ major effects. Moderate and major effects were more common in those over 20
KANSAS JOURNAL of MEDICINE 2017 POISON CONTROL CENTER REPORT

continued.

years of age and in those with intentional encounters. More serious outcomes were related to single-substance pharmaceutical exposures, accounting for $25 \%(n=4)$ of the fatalities. Table 10 lists all medical outcomes by age and Table 11 lists outcomes by reason for exposure.

Use of decontamination and specific therapies, including antidotal therapy, is detailed in Tables 12a and 12b. Sixteen deaths were reported to the PCC in 2017 (Table 13). Fourteen deaths involved adult patients. Twelve deaths involved intentional exposures.

Table 14 compares key statistics from 2015, 2016, and 2017. While total number of exposures declined in 2017 compared to 2016, calls from health care facilities, moderate or major outcomes, and deaths continued to increase.

Table 9. Management site of human exposures.

\begin{tabular}{|c|c|c|}
\hline Site of Management & $\mathbf{N}$ & $\%$ \\
\hline \multicolumn{3}{|l|}{ Healthcare facility } \\
\hline Treated/evaluated and released & 3,131 & 15.4 \\
\hline Admitted to critical care unit & 1,449 & 7.2 \\
\hline Admitted to noncritical care unit & 719 & 3.6 \\
\hline Admitted to psychiatric facility & 481 & 2.4 \\
\hline Patient lost to follow-up/left AMA & 202 & 1.0 \\
\hline Healthcare Facility Subtotal & 5,982 & 29.5 \\
\hline Non-healthcare facility & 14,073 & 69.4 \\
\hline Other & 28 & 0.1 \\
\hline Refused referral & 175 & 0.9 \\
\hline Unknown & 20 & 0.1 \\
\hline Total & 20,278 & 100.0 \\
\hline
\end{tabular}

\section{DISCUSSION}

The University of Kansas Health System Poison Control Center has been in operation for 36 years and receives over 21,000 calls per year. The PCC is an integral part of the emergency medical response, public health, and health care facilities in Kansas. Childhood poisonings, both unintentional and intentional, are a major focus, with calls for patients under 19 years of age accounting for approximately twothirds of all exposures.

The PCC statistics reflect the trends seen nationally. ${ }^{1}$ In 2017, 2,607,413 encounters were logged by poison control centers nationwide, including 2,115,186 human exposures. Total encounters showed a $3.79 \%$ decline from 2016 but health care facility human exposure cases increased by $3.06 \%$. More serious outcomes (moderate, major, or death) have increased since 2000. Nationwide, the five substance classes most frequently involved in adult exposures were analgesics, sedative/hypnotics/antipsychotics, antidepressants, cardiovascular drugs, and cleaning substances (household), while the top five most common exposures in children age five years or less were cosmetics/ personal care products, household cleaning substances, analgesics, foreign bodies/toys/miscellaneous, and topical preparations. There were 2,682 exposure related fatalities reported nationwide in 2017, an increase of 1,190 deaths from $2016 .{ }^{2}$ 
KANSAS JOURNAL of MEDICINE

2017 POISON CONTROL CENTER REPORT

continued.

Table 10. Medical outcome of human exposure cases by patient age.

\begin{tabular}{|c|c|c|c|c|c|c|c|c|c|c|c|c|c|c|c|c|}
\hline \multirow[b]{2}{*}{ Outcome } & \multicolumn{2}{|c|}{$\leq 5$ Years } & \multicolumn{2}{|c|}{6 - 12 Years } & \multicolumn{2}{|c|}{13 - 19 Years } & \multicolumn{2}{|c|}{$\geq 20$ Years } & \multicolumn{2}{|c|}{$\begin{array}{l}\text { Unknown } \\
\text { Child }\end{array}$} & \multicolumn{2}{|c|}{$\begin{array}{c}\text { Unknown } \\
\text { Adult }\end{array}$} & \multicolumn{2}{|c|}{$\begin{array}{c}\text { Unknown } \\
\text { Age }\end{array}$} & \multicolumn{2}{|c|}{ Total } \\
\hline & $\mathbf{N}$ & $\%$ & $\mathbf{N}$ & $\%$ & $\mathbf{N}$ & $\%$ & $\mathbf{N}$ & $\%$ & $\mathbf{N}$ & $\%$ & $\mathbf{N}$ & $\%$ & $\mathbf{N}$ & $\%$ & $\mathbf{N}$ & $\%$ \\
\hline No effect & 3,843 & 36.93 & 280 & 23.89 & 477 & 26.02 & 1,112 & 16.55 & 1 & 12.50 & 6 & 6.38 & 22 & 45.8 & 5,741 & 28.31 \\
\hline $\begin{array}{l}\text { Minor } \\
\text { effect }\end{array}$ & 1,137 & 10.93 & 232 & 19.80 & 562 & 30.66 & 1,733 & 25.80 & 1 & 12.50 & 26 & 27.66 & 2 & 4.2 & 3,693 & 18.21 \\
\hline $\begin{array}{l}\text { Moderate } \\
\text { effect }\end{array}$ & 122 & 1.17 & 37 & 3.16 & 371 & 20.24 & 1,206 & 17.95 & 0 & 0.00 & 3 & 3.19 & 0 & 0.0 & 1,739 & 8.58 \\
\hline $\begin{array}{l}\text { Major } \\
\text { effect }\end{array}$ & 15 & 0.14 & 8 & 0.68 & 51 & 2.78 & 357 & 5.31 & 0 & 0.00 & 0 & 0.00 & 0 & 0.0 & 431 & 2.13 \\
\hline Death & 0 & 0.00 & 1 & 0.09 & 1 & 0.05 & 14 & 0.21 & 0 & 0.00 & 0 & 0.00 & 0 & 0.0 & 16 & 0.08 \\
\hline $\begin{array}{l}\text { No } \\
\text { follow-up, } \\
\text { nontoxic }\end{array}$ & 381 & 3.66 & 34 & 2.90 & 13 & 0.71 & 30 & 0.45 & 0 & 0.00 & 3 & 3.19 & 0 & 0.0 & 461 & 2.27 \\
\hline $\begin{array}{l}\text { No } \\
\text { follow-up, } \\
\text { minimal } \\
\text { toxicity }\end{array}$ & 4,566 & 43.88 & 511 & 43.60 & 263 & 14.35 & 1,709 & 25.44 & 4 & 50.00 & 38 & 40.43 & 8 & 16.7 & 7,099 & 35.01 \\
\hline $\begin{array}{l}\text { No } \\
\text { follow-up, } \\
\text { potentially } \\
\text { toxic }\end{array}$ & 240 & 2.31 & 41 & 3.50 & 69 & 3.76 & 267 & 3.97 & 2 & 25.00 & 15 & 15.96 & 14 & 29.2 & 648 & 3.20 \\
\hline $\begin{array}{l}\text { Unrelated } \\
\text { effect }\end{array}$ & 101 & 0.97 & 28 & 2.39 & 26 & 1.42 & 290 & 4.32 & 0 & 0.00 & 3 & 3.19 & 2 & 4.2 & 450 & 2.22 \\
\hline $\begin{array}{l}\text { Death, } \\
\text { indirect } \\
\text { report }\end{array}$ & 0 & 0.00 & 0 & 0.00 & 0 & 0.00 & 0 & 0.00 & 0 & 0.00 & 0 & 0.00 & 0 & 0.0 & 0 & 0.00 \\
\hline Total & 10,405 & 100.00 & 1,172 & 100.00 & 1,833 & 100.00 & 6,718 & 100.00 & 8 & 100.00 & 94 & 100.00 & 48 & 100.00 & 20,278 & 100.00 \\
\hline
\end{tabular}

Table 11. Medical outcome by reason for exposure in human exposures.

\begin{tabular}{|c|c|c|c|c|c|c|c|c|c|c|c|c|}
\hline \multirow[b]{2}{*}{ Outcome } & \multicolumn{2}{|c|}{ Unintentional } & \multicolumn{2}{|c|}{ Intentional } & \multicolumn{2}{|c|}{ Other } & \multicolumn{2}{|c|}{ Adverse Reaction } & \multicolumn{2}{|c|}{ Unknown } & \multicolumn{2}{|c|}{ Total } \\
\hline & $\mathbf{N}$ & $\%$ & $\mathbf{N}$ & $\%$ & $\mathbf{N}$ & $\%$ & $\mathbf{N}$ & $\%$ & $\mathbf{N}$ & $\%$ & $\mathbf{N}$ & $\%$ \\
\hline Death & 1 & 0.01 & 13 & 0.34 & 0 & 0.00 & 0 & 0.00 & 2 & 2.25 & 16 & 0.08 \\
\hline $\begin{array}{l}\text { Death, indirect } \\
\text { report }\end{array}$ & 0 & 0.00 & 0 & 0.00 & 0 & 0.00 & 0 & 0.00 & 0 & 0.00 & 0 & 0.00 \\
\hline Major effect & 67 & 0.42 & 339 & 8.88 & 3 & 3.70 & 7 & 2.04 & 15 & 16.85 & 431 & 2.13 \\
\hline Minor effect & 2,455 & 15.39 & 1,107 & 28.99 & 22 & 27.16 & 100 & 29.15 & 9 & 10.11 & 3,693 & 18.21 \\
\hline $\begin{array}{l}\text { Moderate } \\
\text { effect }\end{array}$ & 537 & 3.37 & 1,128 & 29.54 & 9 & 11.11 & 45 & 13.12 & 20 & 22.47 & 1,739 & 8.58 \\
\hline No effect & 4,864 & 30.50 & 842 & 22.05 & 13 & 16.05 & 15 & 4.37 & 7 & 7.87 & 5,741 & 28.31 \\
\hline $\begin{array}{l}\text { No follow-up, } \\
\text { nontoxic }\end{array}$ & 443 & 2.78 & 16 & 0.42 & 1 & 1.23 & 1 & 0.29 & 0 & 0.00 & 461 & 2.27 \\
\hline $\begin{array}{l}\text { No follow-up, } \\
\text { minimal } \\
\text { toxicity }\end{array}$ & 6,810 & 42.70 & 172 & 4.50 & 14 & 17.28 & 90 & 26.24 & 13 & 14.61 & 7,099 & 35.01 \\
\hline $\begin{array}{l}\text { No follow-up, } \\
\text { potentially } \\
\text { toxic }\end{array}$ & 445 & 2.79 & 162 & 4.24 & 10 & 12.35 & 18 & 5.25 & 13 & 14.61 & 648 & 3.20 \\
\hline $\begin{array}{l}\text { Unrelated } \\
\text { effect }\end{array}$ & 325 & 2.04 & 39 & 1.02 & 9 & 11.11 & 67 & 19.53 & 10 & 11.24 & 450 & 2.22 \\
\hline Total & 15,947 & 100.00 & 3,818 & 100.00 & 81 & 100.00 & 343 & 100.00 & 89 & 100.00 & 20,278 & 100.00 \\
\hline
\end{tabular}




\section{KANSAS JOURNAL of MEDICINE}

2017 POISON CONTROL CENTER REPORT

\section{continued.}

Table 12a. Decontamination provided in human exposures by age.

\begin{tabular}{|c|c|c|c|c|c|c|c|c|}
\hline Decontamination & $\leq 5$ Years & 6 - 12 Years & 13 - 19 Years & $\geq 20$ Years & $\begin{array}{c}\text { Unknown } \\
\text { Child }\end{array}$ & $\begin{array}{c}\text { Unknown } \\
\text { Adult }\end{array}$ & $\begin{array}{c}\text { Unknown } \\
\text { Age }\end{array}$ & Total \\
\hline Cathartic & 1 & 2 & 18 & 16 & 0 & 0 & 0 & 37 \\
\hline Charcoal, multiple doses & 2 & 0 & 9 & 8 & 0 & 0 & 0 & 19 \\
\hline Charcoal, single dose & 55 & 11 & 153 & 192 & 0 & 0 & 0 & 411 \\
\hline Dilute/irrigate/wash & 7,870 & 754 & 444 & 2,559 & 2 & 38 & 3 & 11,670 \\
\hline Food/snack & 1,589 & 140 & 67 & 355 & 1 & 4 & 0 & 2,156 \\
\hline Fresh air & 105 & 41 & 60 & 442 & 1 & 18 & 22 & 689 \\
\hline Lavage & 0 & 0 & 1 & 5 & 0 & 0 & 0 & 6 \\
\hline Other emetic & 61 & 6 & 13 & 32 & 0 & 0 & 0 & 112 \\
\hline Whole bowel irrigation & 1 & 1 & 3 & 10 & 0 & 0 & 0 & 15 \\
\hline
\end{tabular}


Table 12b. Therapy provided in human exposures by age.

\begin{tabular}{|c|c|c|c|c|c|c|c|c|}
\hline Therapy & $\leq 5$ Years & 6 - 12 Years & 13 - 19 Years & $\geq 20$ Years & Unknown Child & Unknown Adult & Unknown Age & Total \\
\hline Alkalinization & 2 & 1 & 48 & 140 & 0 & 0 & 0 & 191 \\
\hline Antiarrhythmic & 1 & 0 & 1 & 9 & 0 & 0 & 0 & 11 \\
\hline Antibiotics & 23 & 8 & 14 & 159 & 0 & 0 & 0 & 204 \\
\hline Anticonvulsants & 0 & 1 & 1 & 5 & 0 & 0 & 0 & 7 \\
\hline Antiemetics & 22 & 5 & 115 & 231 & 0 & 0 & 0 & 373 \\
\hline Antihistamines & 23 & 14 & 11 & 76 & 0 & 0 & 0 & 125 \\
\hline Antihypertensives & 1 & 1 & 2 & 20 & 0 & 0 & 0 & 24 \\
\hline Antivenin/antitoxin & 0 & 1 & 2 & 6 & 0 & 0 & 0 & 9 \\
\hline Antivenin (fab fragment) & 2 & 3 & 1 & 18 & 0 & 0 & 0 & 24 \\
\hline Atropine & 0 & 1 & 1 & 15 & 0 & 0 & 0 & 17 \\
\hline Benzodiazepines & 18 & 5 & 80 & 296 & 0 & 0 & 0 & 399 \\
\hline Bronchodilators & 7 & 4 & 9 & 63 & 0 & 0 & 0 & 83 \\
\hline Calcium & 156 & 8 & 4 & 26 & 0 & 0 & 0 & 194 \\
\hline Cardioversion & 0 & 0 & 0 & 2 & 0 & 0 & 0 & 2 \\
\hline $\mathrm{CPR}$ & 0 & 0 & 0 & 7 & 0 & 0 & 0 & 7 \\
\hline Deferoxamine & 0 & 0 & 1 & 1 & 0 & 0 & 0 & 2 \\
\hline Fab fragments & 1 & 0 & 4 & 4 & 0 & 0 & 0 & 9 \\
\hline Fluids, IV & 75 & 24 & 558 & 1,502 & 0 & 0 & 0 & 2,159 \\
\hline Flumazenil & 0 & 0 & 7 & 43 & 0 & 0 & 0 & 50 \\
\hline Folate & 0 & 0 & 0 & 2 & 0 & 0 & 0 & 2 \\
\hline Fomepizole & 0 & 1 & 0 & 17 & 0 & 0 & 0 & 18 \\
\hline Glucagon & 0 & 0 & 1 & 25 & 0 & 0 & 0 & 26 \\
\hline Glucose, $>5 \%$ & 0 & 0 & 7 & 45 & 0 & 0 & 0 & 52 \\
\hline Hemodialysis & 0 & 0 & 0 & 22 & 0 & 0 & 0 & 22 \\
\hline Hemoperfusion & 0 & 0 & 0 & 1 & 0 & 0 & 0 & 1 \\
\hline Hydroxocobalamin & 0 & 0 & 0 & 4 & 0 & 0 & 0 & 4 \\
\hline Hyperbaric oxygen & 0 & 2 & 1 & 6 & 0 & 0 & 0 & 9 \\
\hline Insulin & 0 & 0 & 1 & 25 & 0 & 0 & 0 & 26 \\
\hline Intubation & 8 & 2 & 32 & 199 & 0 & 0 & 0 & 241 \\
\hline Methylene blue & 0 & 0 & 0 & 3 & 0 & 0 & 0 & 3 \\
\hline NAC, IV & 0 & 2 & 85 & 157 & 0 & 0 & 0 & 244 \\
\hline NAC, PO & 0 & 0 & 25 & 17 & 0 & 0 & 0 & 42 \\
\hline Naloxone & 10 & 1 & 17 & 153 & 0 & 0 & 0 & 181 \\
\hline Neuromuscular blocker & 0 & 0 & 1 & 14 & 0 & 0 & 0 & 15 \\
\hline Octreotide & 0 & 0 & 0 & 5 & 0 & 0 & 0 & 5 \\
\hline Oxygen & 23 & 11 & 64 & 421 & 0 & 0 & 0 & 519 \\
\hline Physostigmine & 0 & 0 & 2 & 8 & 0 & 0 & 0 & 10 \\
\hline Phytonadione & 0 & 0 & 2 & 7 & 0 & 0 & 0 & 9 \\
\hline Sedation (other) & 9 & 3 & 31 & 173 & 0 & 0 & 0 & 216 \\
\hline Sodium thiosulfate & 0 & 0 & 0 & 1 & 0 & 0 & 0 & 1 \\
\hline Steroids & 13 & 3 & 4 & 67 & 0 & 1 & 0 & 88 \\
\hline Succimer & 0 & 0 & 0 & 4 & 0 & 0 & 0 & 4 \\
\hline Vasopressors & 1 & 1 & 5 & 79 & 0 & 0 & 0 & 86 \\
\hline Ventilator & 8 & 2 & 30 & 195 & 0 & 0 & 0 & 235 \\
\hline Other & 66 & 25 & 96 & 444 & 0 & 2 & 0 & 633 \\
\hline
\end{tabular}


Table 13. Details on deaths and exposure related fatalities.

\begin{tabular}{|c|c|c|c|c|c|c|c|}
\hline Non-Pharmaceutical Exposures & Age, Gender & Substances & Substance Rank & Cause Rank & Chronicity & Route & Reason $^{* * *}$ \\
\hline Cleaning substances (household) & 58 years, Male & Drain Cleaner & 1 & 1 & Acute & Ingestion & Int. - suicide \\
\hline Fumes/gases/vapors & 64 years, Male & Carbon Monoxide & 1 & 1 & Acute & Inhalation & Unint. - Env. \\
\hline \multirow[t]{3}{*}{ Heavy metals } & 59 years, Male & Arsenic & 1 & 1 & Acute & Ingestion & Int. - suicide \\
\hline & & Benzene & 2 & 2 & & & \\
\hline & & Toluene & 3 & 3 & & & \\
\hline \multicolumn{8}{|l|}{ Pharmaceutical Exposures } \\
\hline \multirow[t]{6}{*}{ Analgesics } & 32 years, Female & Acetaminophen & 1 & 1 & Acute & Ingestion & Int. - suicide \\
\hline & 56 years, Male & Acetaminophen/Oxycodone & 1 & 1 & Acute & Ingestion & Int. - suicide \\
\hline & & Alprazolam* & 2 & 2 & & & \\
\hline & & Drug, unknown & 3 & 3 & & & \\
\hline & 76 years, Female & Acetaminophen & 1 & 1 & Acute & Ingestion & Int. - suicide \\
\hline & 76 years, Male & Salicylate & 1 & 1 & Acute & Ingestion & Int. - suicide \\
\hline \multirow[t]{15}{*}{ Cardiovascular drugs } & 9 years, Female & Clonidine & 1 & 1 & Acute & Ingestion & Unknown \\
\hline & & Cationic detergent & 2 & 2 & & & \\
\hline & & Cyproheptadine & 3 & 3 & & & \\
\hline & & Methylphenidate & 4 & 4 & & & \\
\hline & & Alpha-adrenergic blocker & 5 & 5 & & & \\
\hline & & Desmopressin & 6 & 6 & & & \\
\hline & 26 years, Male & Nebivolol & 1 & 1 & Acute & Ingestion & Int. - suicide \\
\hline & & $\begin{array}{l}\text { Hydrochlorothiazide/ } \\
\text { Losartan }\end{array}$ & 2 & 2 & & & \\
\hline & 57 years, Female & Diltiazem (extended release) & 1 & 1 & Acute & Ingestion & Int. - suicide \\
\hline & & Sotalol & 2 & 2 & & & \\
\hline & & Apixaban & 3 & 3 & & & \\
\hline & & Losartan & 4 & 4 & & & \\
\hline & & Cholecalciferol & 5 & 5 & & & \\
\hline & & Folic Acid & 6 & 6 & & & \\
\hline & & Ethanol & 7 & 7 & & & \\
\hline \multirow[t]{2}{*}{ Dietary supplements/herbals/homeopathic } & 19 years, Male & Piper Methysticum & 1 & 1 & Acute & Ingestion & Int.- abuse \\
\hline & & Sertraline & 2 & 2 & & & \\
\hline \multirow[t]{2}{*}{ Hormones and hormone antagonists } & 31 years, Female & Metformin & 1 & 1 & $\begin{array}{l}\text { Acute on } \\
\text { Chronic }\end{array}$ & Ingestion & Int. - suicide \\
\hline & & Ibuprofen** & 2 & 2 & & & \\
\hline \multirow[t]{4}{*}{ Miscellaneous drugs } & 50 years, Female & Ropinirole & 1 & 1 & $\begin{array}{l}\text { Acute on } \\
\text { Chronic }\end{array}$ & Ingestion & Int. - suicide \\
\hline & & Clopidogrel & 2 & 2 & & & \\
\hline & & Alprazolam* & 3 & 3 & & & \\
\hline & & Ethanol & 4 & 4 & & & \\
\hline \multirow[t]{3}{*}{ Sedative/hypnotics/antipsychotics } & 44 years, Male & Ziprasidone & 1 & 1 & $\begin{array}{l}\text { Acute on } \\
\text { Chronic } \\
\end{array}$ & Ingestion & Int. - suicide \\
\hline & & $\begin{array}{c}\text { Valproic Acid } \\
\text { (extended release) }\end{array}$ & 2 & 2 & & & \\
\hline & & $\begin{array}{l}\text { Acetaminophen// } \\
\text { hydrocodone }\end{array}$ & 3 & 3 & & & \\
\hline \multirow[t]{3}{*}{ Stimulants and street drugs } & 26 years, Male & Methamphetamine & 1 & 1 & Acute & Unknown & Unknown \\
\hline & & Amphetamine & 2 & 2 & & & \\
\hline & 28 years, Male & Methamphetamine & 1 & 1 & Acute & Ingestion & $\begin{array}{l}\text { Int. - } \\
\text { unknown } \\
\text { reason }\end{array}$ \\
\hline
\end{tabular}


KANSAS JOURNAL of MEDICINE

2017 POISON CONTROL CENTER REPORT

continued.

Table 14. 2015 to 2017 comparison of select statistics.

\begin{tabular}{|l|c|c|c|}
\hline & $\mathbf{2 0 1 5}$ & $\mathbf{2 0 1 6}$ & $\mathbf{2 0 1 7}$ \\
\hline Total cases & 20,109 & 21,965 & 21,431 \\
\hline Calls from HCF & 4,267 & 4,514 & 4,892 \\
\hline $\begin{array}{l}\text { Moderate or major } \\
\text { outcomes }\end{array}$ & 1,688 & 1,971 & 2,170 \\
\hline Deaths & 13 & 15 & 16 \\
\hline
\end{tabular}

The ongoing importance of the PCC is reflected in increasing trends in rates of poisonings and overdoses that have reached epidemic proportions in some cases. The PCC saw an increase in number of calls from health care facilities, cases with moderate or major medical outcomes, and deaths in 2017 compared to 2016. This is consistent with literature that notes in the United States a 9.6\% increase in drug overdose deaths in 2017 compared to $2016 .{ }^{3}$ A vast majority of these deaths are related to opioids and one study projects an increase of opioid related deaths from 42,200 in 2016 to over 80,000 per year by $2025 .{ }^{4}$ Adolescent intentional overdose also impacts the increase in morbidity. Several studies have documented increasing numbers of adolescent overdose with subsequent increase in morbidity. ${ }^{5-7}$

Reporting exposures to the PCC is voluntary and the PCC is not contacted for all poisonings in the state of Kansas. Furthermore, in most cases, there is no objective confirmation of exposure. These limitations should be noted when interpreting PCC data.

\section{CONCLUSION}

The results of the 2017 University of Kansas Health System Poison Control annual report demonstrated that the center continues to receive calls from the entire state of Kansas totaling over 20,000 human exposures per year. While pediatric exposures remain the most common, there is an increasing number of calls from health care facilities and for cases with serious outcomes. The experience of the PCC reflects the national trends of increasing morbidity and mortality associated with overdoses and other exposures. This report demonstrated the continued value of the PCC to both the public and to health care providers in the state of Kansas.

\section{ACKNOWLEDGEMENTS}

The authors acknowledge the efforts of the Poison Control Center staff: Tama Sawyer, Anne Marie Banks, Amber Ashworth, Mike McKinney, Kathy White, Anita Farris, Mark Stallbaumer, Bobbie Jean Wainscott, and Connor Bowman.

\section{REFERENCES}

${ }^{1}$ Gummin DD, Mowry JB, Spyker DA, Brooks DE, Osterthaler KM, Banner W. 2017 Annual Report of the American Association of Poison Control Centers' National Poison Data System (NPDS): 35th Annual Report. Clin Toxicol (Phila) 2018; 56(12):1213-1415. PMID: 30576252.

2 Gummin DD, Mowry JB, Spyker DA, Brooks DE, Fraser MO, Banner W. 2016 Annual Report of the American Association of Poison Control Centers' National Poison Data System (NPDS): 34th Annual Report. Clin Toxicol (Phila) 2017; 55(10):1072-1252. PMID: 29185815.

${ }^{3}$ Hedegaard H, Miniño AM, Warner M. Drug overdose deaths in the United States, 1999-2017. NCHS Data Brief 2018; (329):1-8. PMID: 30500323.

${ }^{4}$ Chen Q, Larochelle MR, Weaver DT, et al. Prevention of prescription opioid misuse and projected overdose deaths in the United States. JAMA Netw Open 2019; 2(2):el87621. PMID: 30707224.

${ }^{5}$ Pringle K, Caupp S, Shi J, et al. Analysis of intentional drug poisonings using Ohio Poison Control Center Data, 2002-2014. Clin Toxicol (Phila) 2017; 55(7):652-658. PMID: 28393553.

${ }^{6}$ Froberg BA, Morton SJ, Mowry JB, Rusyniak DE. Temporal and geospatial trends of adolescent intentional overdoses with suspected suicidal intent reported to a state poison control center. Clin Toxicol (Phila) 2019; 30:1-8. PMID: 30696297.

7 Sheridan DC, Hendrickson RG, Lin AL, Fu R, Horowitz BZ. Adolescent suicidal ingestion: National trends over a decade. J Adolesc Health 2017; 60(2):191-195. PMID: 27889404.

Keywords: drug overdose, poisoning, ingestion, toxicology, antidotes

*Supplemental appendix available at journals.ku.edu/kjm.* 